\title{
Surface-expressed enolases of Plasmodium and other pathogens
}

\author{
Anil Kumar Ghosh, Marcelo Jacobs-Lorena/ ${ }^{+}$ \\ Department of Molecular Microbiology and Immunology and Malaria Research Institute, \\ Johns Hopkins Bloomberg School of Public Health, 615 N Wolfe Street, Baltimore, 21205 MD, USA
}

Enolase is the eighth enzyme in the glycolytic pathway, a reaction that generates ATP from phosphoenol pyruvate in cytosolic compartments. Enolase is essential, especially for organisms devoid of the Krebs cycle that depend solely on glycolysis for energy. Interestingly, enolase appears to serve a separate function in some organisms, in that it is also exported to the cell surface via a poorly understood mechanism. In these organisms, surface enolase assists in the invasion of their host cells by binding plasminogen, an abundant plasma protease precursor. Binding is mediated by the interaction between a lysine motif of enolase with Kringle domains of plasminogen. The bound plasminogen is then cleaved by specific proteases to generate active plasmin. Plasmin is a potent serine protease that is thought to function in the degradation of the extracellular matrix surrounding the targeted host cell, thereby facilitating pathogen invasion. Recent work revealed that the malaria parasite Plasmodium also expresses surface enolase, and that this feature may be essential for completion of its life cycle. The therapeutic potential of targeting surface enolases of pathogens is discussed.

Key words: Plasmodium - cell surface enolase - pathogenicity

The global burden of parasitic diseases is a problem of enormous proportions, especially in developing countries (Table I). The highest burden is inflicted by malaria, with the number of people at risk approaching two billion and expected to keep increasing (Snow et al. 2005). Malaria is most severe in the sub-Saharan Africa where a child dies every $40 \mathrm{~s}$ and imposes an annual economic burden of $\$ 12$ billion (Rugemalila et al. 2007).

To survive, obligate intracellular pathogens need to invade their host tissues. Within their target cells the microbes are safe to propagate and prepare for transmission to the next host. Pathogens from bacteria to helminths have developed a variety of mechanisms to invade host tissues. Several bacteria develop fimbria or different types of pilli, for the initial contact with their host cells. Other protozoa develop pseudopodia, which are undulating membranous flagella, for increased penetration efficiency. Helminthic parasites use a variety of cutting appendages and suckers for anchoring and invasion. In addition to the above-mentioned structural modifications, several biochemical arsenals have developed during the course of evolution, including adhesion molecules and cellular receptors (Pizarro-Cerdá \& Cossart 2006). A number of pathogenic organisms display specialized proteins on their cell surface to assist in invasion, one of the best characterized being the glycolytic enzyme enolase. Cell surface enolase efficiently captures plasminogen from the surrounding environment which is subsequently converted into plasmin, a strong serine protease that facilitates the invasion process (Lot-

Financial support: NIH (AI031478), JHMRI, Bloomberg Family Foundation + Corresponding author: mlorena@jhsph.edu

Received 22 April 2011

Accepted 23 May 2011 tenberg et al. 1994, Ehinger et al. 2004). Here we review what is known about the role played by surface enolase in Plasmodium and other pathogens.

Enolase and plasminogen - Enolase is an ancient and ubiquitous metalloenzyme. It catalyzes the reversible dehydration of 2-phosphoD-glycerate into P-enolpyruvate during glycolysis. Eubacteria and archaebacteria have a single enolase gene and therefore multiple molecular forms (isoforms) are not observed. Overall, enolase sequences are highly conserved, showing roughly $40 \%$ amino acid identity across eukaryotes, eubacteria and archaebacteria although a large number of insertion and deletions occur across phyla. Some of the insertions and deletions have been shown to be highly plastic (Keeling et al. 2004). In yeast and most higher organisms, enolase subunits assemble into dimers while in some eubacteria (e.g. Bacillus subtilis and Thermotoga maritima) the protein is octameric. Plasmodium has a single enolase gene in chromosome $10.92 \% \mathrm{~A}+\mathrm{T}$ content (Read et al. 1994). The protein has 446 amino acids residues $(48.7 \mathrm{kDa})$, similar in length to enolases from other eukaryotes. Plasmodium enolase has a common ancestry with apicomplaxan and plants and may have derived from their plastid endosymbionts (Read et al. 1994, Hannaert et al. 2000). Plasmodium enolase carries a EWGWS pentapeptide insertion (residues 104-108) that is immunogenic and may be exposed on the surface of the parasites (Vora et al. 2009). It is unclear how enolase is exported to the surface of the parasite. The absence of a signal peptide indicates that enolase does not transit through the endoplasmic reticulum but it has been suggested that enolase enters the classical secretory pathway by a previously unidentified mechanism (López-Villar et al. 2006).

Plasminogen is a $92 \mathrm{kDa}$ proenzyme that requires cleavage at its C-terminal region (Ponting et al. 1992) for activity. Activation of plasminogen into active plasmin involves cleavage of the $\mathrm{Arg}_{561}-\mathrm{Val}_{562}$ bond yielding an N- 
terminal A (heavy) chain which remains linked by two disulphide bonds to the smaller C-terminal B (light) chain. The $\mathrm{B}$ chain contains the protease-active residues (Ponting et al. 1992). The plasminogen $\mathrm{N}$-terminal region contains five 80-amino-acids-long Kringle domains. Plasminogen binds strongly and specifically to surface enolase via interaction of its Kringle domains with the C-terminal or internal lysine motifs of enolase (Derbise et al. 2004).

Plasmodium surface enolase and midgut invasion For transmission to occur, Plasmodium needs to complete a complex life cycle within its mosquito vector. The cycle begins with the rapid differentiation of gametocytes acquired with the infected blood meal into male and female gametes, followed by fertilization and differentiation of the resulting zygotes into motile ookinetes. The ookinete must then cross two physical barriers: the peritrophic matrix (Shao et al. 2001) and the midgut epithelium (Ghosh et al. 2000). We recently found that enolase is exported to the surface of ookinetes but not of gametocytes, gametes and zygotes (AK Ghosh et al., unpublished observations). Ookinete surface enolase appears to perform two separate functions: (i) bind to a mosquito midgut epithelial receptor for invasion of the midgut and (ii) recruit plasminogen (present in the ingested blood) via enolase's internal lysine motif (DKSLVK). Recruitment of plasminogen to the ookinete surface is essential, as depletion of plasminogen from the blood meal completely aborts parasite development in the mosquito. Replenishment of the plasminogen-depleted blood with recombinant wild type plasminogen restores mosquito infection, while addition of a plasminogen point mutant in plasmin's active site does not restore infection (AK Ghosh et al., unpublished observations). These results suggest that not only is plasminogen required, but that its conversion to active plasmin is essential. We speculate that plasmin functions in degradation of the extracellular matrix (glycocalyx) that covers the mosquito midgut epithelium (Zieler et al. 2000) (A in Figure).
Sato et al. (2000) found that patients infected with Plasmodium falciparum and Plasmodium vivax carry high anti-enolase antibody titres and concluded that enolase could be used for the immunodiagnosis of malaria. Roth et al. (1988) found that the total amount of enolase in parasitized red blood cells (RBCs) increases about 15 -fold during infection. Recently, we found that enolase also inserts on the surface of the RBCs infected with the human parasite $P$. falciparum (unpublished observation). The role played by this RBC surface enolase is not known.

Enolase-mediated cell invasion is a common theme among pathogenic microorganism - A brief summary of the occurrence of cell surface enolase across different phyla and their potential role in pathogenesis is provided in Table II. Candida albicans can cause superficial infections of the oral and vaginal mucosa and in some cases causes life-threatening disseminated disease (Cutler 1991). C. albicans may also invade the central nervous system resulting in devastating meningitis (Zhang et al. 1999). To reach the central nervous system C. albicans must cross the blood-brain barrier by traversing the endothelial lining of the brain capillaries. It was found that Candida binds both plasminogen and plasmin in a specific and saturable manner. Plasminogen binding to the Candida surface, which is mediated by surface enolase, greatly enhances its affinity for streptokinase, an enzyme involved in plasminogen activation to plasmin. The net result is enhanced fibrinolysis and increased ability to cross an in vitro blood-brain barrier system (Jong et al. 2003) (B in Figure).

Streptococcus pneumoniae is the causative agent of bacterial meningitis. S. pneumoniae can degrade extracellular matrix components thus breaching the bloodbrain barrier and penetrating brain tissue to cause severe disease. The $S$. pneumoniae enolase gene encodes a $47 \mathrm{kDa}$ protein with no signal sequence or transmembrane domain and as for other pathogens it is not known how this protein reaches the cell surface and remains attached

TABLE I

Public health importance of selected human parasitic infections

\begin{tabular}{lcccc}
\hline Disease & $\begin{array}{c}\text { Population } \\
\text { at risk (m) }\end{array}$ & $\begin{array}{c}\text { Endemic } \\
\text { countries (n) }\end{array}$ & $\begin{array}{c}\text { Cases/year } \\
(\mathrm{m})\end{array}$ & $\begin{array}{c}\text { Mortality } \\
\text { (deaths/year x 1,000) }\end{array}$ \\
\hline Malaria & 2,000 & 90 & $300-500$ & 1,080 \\
Leishmaniasis & 350 & 82 & 12 & 41 \\
Chagas disease & 90 & 19 & 16 & 21 \\
Trypanosomiasis & 50 & 36 & $20,000-300,000$ & 50 \\
Entamoeba & - & - & 500 & $40-100$ \\
Giardia & - & - & 200 & - \\
Lymphatic filariasis & 750 & 65 & 119 & NDM \\
Schistosomiasis & $500-600$ & 74 & 200 & 11 \\
Fascioliasis & 180.25 & 8 & 17.6 & - \\
Onchocerciasis & 122 & 34 & 15 & NDM \\
Taeniasis & 40 & - & -
\end{tabular}

adapted from Molyneux (2006) and Hotez et al. (2006). m: million; NDM: no direct mortality. 
to it. The S. pneumoniae enolase protein is octameric and has two plasminogen-binding motifs, one at the C-terminal end and the other internal, from amino acid 248-256 (Ehinger et al. 2004). In an elegant experiment where reconstituted basement membrane (Matrigel) was used to test the penetration process, it was observed that bacteria only invade the basement membrane when in medium containing plasminogen and when surface bound plasminogen was cleaved by tissue plasminogen activator (tPA). In the absence of plasminogen bacteria could not penetrate even after $21 \mathrm{~h}$ incubation. The results suggest a potential role of surface-associated plasminogen in bacterial penetration of basement membranes and extracellular matrices (Eberhard et al. 1999).

Staphylococcus aureus is a highly invasive bacterium that causes a large number of the hospital infections throughout the world, having a major impact on human morbidity and mortality. S. aureus can colonize the mucosa of airways and also has a propensity for targeting bone tissues, thereby causing bone disease, especially osteomyelitis (Wheat 1985). The bacterium produces a $52 \mathrm{kDa}$ protein with high homology with enolase that is transported to the cell wall surface by an unknown mechanism. This $52 \mathrm{kDa}$ protein binds to laminin and might function by first allowing $S$. aureus to adhere to the extracellular matrix, followed by plasminogen activation and laminin degradation around the penetration area (Carneiro et al. 2004).

Streptococcus pyogenes is responsible for a wide variety of human disease that range from infections of the throat (pharyngitis), skin (impetigo) and degeneration of underlying tissue (necrotizing fasciitis or flesheating disease), to a total toxic shock syndrome, rheumatic fever and acute glomerulonephritis (Pancholi \& Fischetti 1998). S. pyogens expresses a protein of about $45 \mathrm{kDa}$ called streptococcal surface enolase on its surface. It bears a C-terminal lysine residue which binds to the Kringle domain of plasminogen thus promoting the invasion process (Derbise et al. 2004).

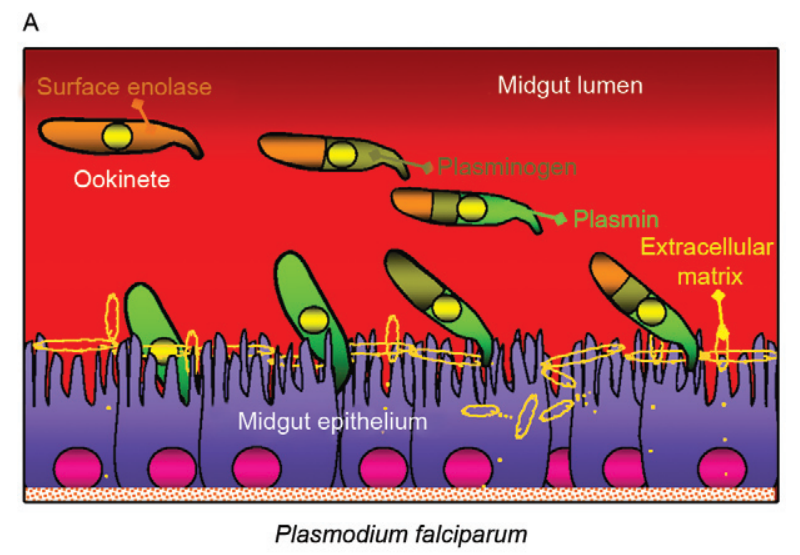

B

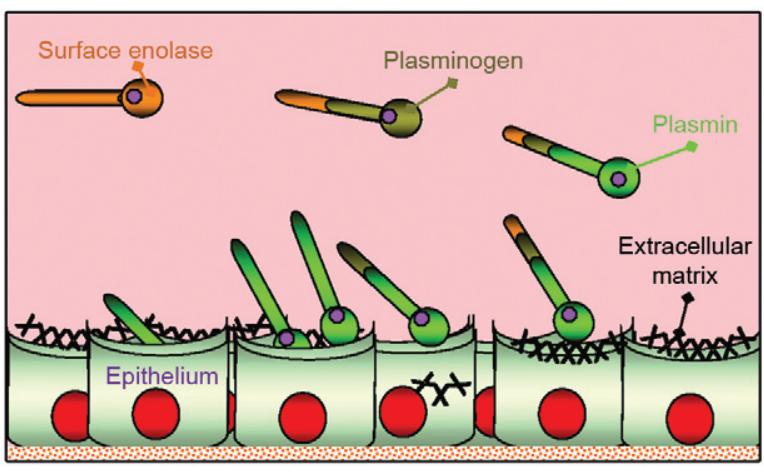

Candida albicans

Model for the role of plasminogen in pathogen invasion of host tissues. Similar mechanisms are hypothesized to operate for apicomplexans (e.g., Plasmodium falciparum) (A), fungi (e.g., Candida albicans) (B) and bacteria (not shown). From left to right: (i) the pathogen exports enolase protein to its surface (burned sienna colour) by an unknown mechanism; (ii) surface enolase captures mammalian plasminogen (olive green) from its environment; (iii) plasminogen is activated into plasmin (grass green), likely by tissue plasminogen activator (not shown); (iv) surface plasmin promotes the degradation of the extracellular matrix. Degradation of this physical barrier promotes invasion of the underlying epithelium.

TABLE II

Enolase of different pathogenic microorganisms

\begin{tabular}{|c|c|c|c|c|c|}
\hline Pathogen & $\begin{array}{c}\text { Cellular } \\
\text { localization }\end{array}$ & Pathogenesis & $\begin{array}{c}\text { Interacting } \\
\text { cell/tissue type }\end{array}$ & $\begin{array}{l}\text { Size } \\
(\mathrm{kDa})\end{array}$ & Reference \\
\hline \multicolumn{6}{|l|}{ Fungi } \\
\hline Candida albicans & $\mathrm{C}$ and $\mathrm{S}$ & Meningitis & Brain & 47 & Jong et al. (2003) \\
\hline \multicolumn{6}{|l|}{ Bacteria } \\
\hline Streptococcus pneumoniae & $\mathrm{C}$ and $\mathrm{S}$ & Pneumonia & Brain & 47 & Eberhard et al. (1999) \\
\hline Staphylococcus aureus & $\mathrm{C}$ and $\mathrm{S}$ & Toxic shock & Systemic & 52 & Carneiro et al. (2004) \\
\hline \multicolumn{6}{|l|}{ Protozoa } \\
\hline Leishmania mexicana & $\mathrm{C}$ and $\mathrm{S}$ & Cutaneous leishmaniosis & Skin & 42 & Quiñones et al. (2007) \\
\hline Plasmodium falciparum & $\mathrm{C}$ and $\mathrm{S}$ & Malaria & $\mathrm{RBC}, \mathrm{mosq}^{a}$ & 47 & Pal-Bhowmick et al. (2007) \\
\hline \multicolumn{6}{|l|}{ Helminth } \\
\hline Onchocerca volvulus & $\mathrm{C}$ and $\mathrm{S}$ & River blindness & Skin, eye & 38 & Jolodar et al. (2003) \\
\hline
\end{tabular}

a: mosquito midgut (unpublished observations); C: cytosolic; RBC: red blood cell; S: surface. 
Leishmania spp are protozoan parasites that are the cause of cutaneous and visceral leishmaniasis. Leishmania mexicana expresses a $47 \mathrm{kDa}$ enolase that localizes mainly to the cytoplasm but a significant proportion is found facing the external surface of the cell (Quiñones et al. 2007). Leishmania surface enolase binds plasminogen (Avilan et al. 2000, Calcagno et al. 2002) and this interaction contributes to the virulence of the parasite (Maldonado et al. 2006). An anti-enolase antibody interferes with plasminogen binding, making this a vaccine candidate antigen.

Human onchocerciasis is caused by the filarial parasite Onchocerca volvulus and is a major cause of skin pathology and eye lesion, including blindness (river blindness). An estimated 18 million people are infected and about 120 million people are at risk of infection mainly in tropical Africa. The parasite is transmitted by female black flies of the genus Simulium. The incubation period in man is about one year. The infected larvae remain localized in the skin and grow into adults producing large numbers of microfilariae that are the form transmitted by black flies and the main cause of disease symptoms. $O$. volvulus encodes an enolase of about $38 \mathrm{kDa}$. The protein was observed to be localized to the afibrillar portion of the body wall muscle and to the uterus. It is not known how the protein is transported to the outer cell surface (Jolodar et al. 2003).

Therapeutic potential of targeting cell surface enolase - Previous mouse infection and in vitro experiments indicated that binding of plasminogen to pneumococci, and in particular to surface-exposed enolase, is important for in vivo virulence and plasmin-mediated degradation of the extracellular matrix (Bergmann et al. 2003, 2005). The presence of anti-pneumococcal antibodies in sera from young children with and without a history of pneumococcal contact has been compared (Adrian et al. 2004). No significant correlation between antibody titres and infection was observed. Since enolase is a conserved glycolytic enzyme, it was concluded that anti-enolase antibodies in young children probably represented cross-reactive epitopes to commensal bacteria (Adrian et al. 2004).

Epitope accessibility on viable bacteria is of critical importance in the development of protective antibodies to bacterial infections. The epitope of the anti-enolase $\mathrm{mAb}$ (245, C-6) was mapped to residues ${ }_{55}$ DKSRYGGLG $_{63}$ in the N-terminal domain of S. pneumoniae enolase (Ehinger et al. 2004). Crystal structure of the protein indicated that these residues are surface-exposed on the enolase octamer.

Immuno-electron microscopy and flow cytometric studies revealed that the abundance of surface-exposed enolase of in vitro-grown pneumococci is relatively low. However, pneumococcal colonization is a multifactorial process which also might involve changes in expression of the enolase protein under in vivo conditions (Kolberg et al. 2006). Despite the low amount of enolase protein on in vitro-grown pneumococci, the efficiency of plasminogen binding is high and this amount is sufficient to contribute to pneumococcal pathogenesis as shown previously by Bergmann et al. (2003) and Kolberg et al. (2006).

Streptococcus suis serotype 2 (SS2) is a porcine and human pathogen that can cause meningitis, endocarditis and septic shock in people who handle swine and its products (Trottier et al. 1991). Mice immunized with anti-recombinant enolase gave rise to a high titre humoral response resulting in an $83 \%$ protection after challenge with the SS2 bacterium (Zhang et al. 2009). Dental caries is a prevalent chronic human infection caused by Streptococcus sorbinas and Streptococcus mutants. When a formulation of recombinant sorbinas enolase in alum was administered to the oral cavity of rats, it elicited a high humoral antibody response while reducing enamel loss and dental caries (Dinis et al. 2009).

Immunofluorescence as well as electron microscopic examinations revealed that $P$. falciparum enolase localizes on the merozoite surface. Mice immunized with recombinant $P$. falciparum enolase showed protection against a challenge with a lethal strain of Plasmodium yoelii suggesting that enolase is a potential protective antigen (Pal-Bhowmick et al. 2007). In a separate set of experiments mice were immunized with recombinant $P$. falciparum enolase and then infected with Plasmodium berghei. Mosquitoes feeding on the infected immunized mice had a significant lower number of oocysts, suggesting that enolase could be used as an antigen for a transmission blocking vaccine (unpublished observations).

Perspectives - The life cycle of pathogens frequently involves crossing of physical barriers such as extracellular matrices and tissue epithelia. This is true for pathogen development in their mammalian as well as invertebrate hosts. Mammals make use of the potent protease plasmin, to regulate its clotting system. Plasminogen, the plasmin precursor, binds to fibrin via its Kringle motifs and this binding increases its susceptibility to activating enzymes such as tPA. It is interesting that such a wide variety of pathogens, from bacteria to multicellular organisms, opted to recruit the plasminogen-plasmin system of their mammalian hosts to their own advantage. An additional challenge for the pathogen is that plasminogen needs to be captured on the pathogen's surface which is devoid of fibrin. To this end, pathogens evolved a mechanism to export one of their metabolic enzymes enolase or a related protein, to the cell surface, as these proteins carry a lysine motif that physically interacts with the Kringle motifs of plasminogen. The genome of these pathogens frequently has a single enolase gene whose protein product has to accomplish two important functions: act (i) as a metabolic enzyme and (ii) as a surface anchor. What mechanisms operate to provide such different functions to the same protein is not known for any organism. After synthesis on polyribosomes, what determines whether the protein (enolase or enolase-like) remains in the cytoplasm or is exported to the surface? As the protein does not have a recognizable signal sequence, by what mechanism(s) does it reach the surface? As the protein does not have a recognizable trans-membrane domain, by what mechanism(s) does it stay attached to the pathogen surface? In any event, it is interesting that such a broad range of organisms (convergently?) evolved the same strategy to capture plasminogen on their surface.

Additional outstanding questions include the mechanisms of plasminogen activation. As plasminogen binding to fibrin makes it more susceptible to its activating enzymes, it is reasonable to speculate that the same is 
true for plasminogen binding to enolase on the pathogen surface. However, not much is known about which enzymes actually catalyze the conversion of plasminogen to plasmin on the pathogen surface. In case of Plasmodium, our laboratory has preliminary evidence that tPA binds to the surface of the mosquito midgut epithelium, suggesting the hypothesis that activation of plasminogen on the ookinete surface actually occurs at the time of ookinete-midgut epithelium contact.

Another outstanding question is the nature of the protein substrates of surface plasmin during tissue invasion. In mammals, the principal activity of plasmin is the degradation of fibrin. However, plasmin also degrades noncollagenous extracellular matrix proteins such as laminin (Carneiro et al. 2004). In an in vitro experiment it was also found that Hemophilus influenzae can degrade laminin and fibronectin (Virkola et al. 1996). What are the substrates that are cleaved by plasmin during pathogen invasion? The answer to this question is of obvious importance as such knowledge could be explored to devise novel approaches for disease control.

\section{ACKNOWLEDGEMENTS}

To Johns Hopkins Malaria Research Institute and P. falciparum core facilities, for help with mosquito rearing and parasite cultures, and to Dr Clive Schiff, for his comments on the epidemiology of parasitic diseases.

\section{REFERENCES}

Adrian PV, Bogaert D, Oprins M, Rapola S, Lahdenkari M, Kilpi T, de Groot R, Käyhty H, Hermans PW 2004. Development of antibodies against pneumococcal proteins alpha-enolase, immunoglobulin A1 protease, streptococcal lipoprotein rotamase A, and putative proteinase maturation protein $\mathrm{A}$ in relation to pneumococcal carriage and otitis media. Vaccine 22: 2737-2742.

Avilan L, Calcagno M, Figuera M, Lemus L, Puig J, Rodriguez AM 2000. Interaction of Leishmania mexicana promastigotes with the plasminogen-plasmin system. Mol Biochem Parasitol 110: 183-193.

Bergmann S, Rohde M, Preissner KT, Hammerschmidt S 2005. The nine residue plasminogen-binding motif of the pneumococcal enolase is the major cofactor of plasmin-mediated degradation of extracellular matrix, dissolution of fibrin and transmigration. Thromb Haemost 94: 304-311.

Bergmann S, Wild D, Diekmann O, Frank R, Bracht D, Chhatwal GS, Hammerschmidt S 2003. Identification of a novel plasmin(ogen)binding motif in surface displayed alpha-enolase of Streptococcus pneumoniae. Mol Microbiol 49: 411-423.

Calcagno M, Avilan L, Colasante C, Berrueta L, Salmen 2002. Interaction of different Leishmania mexicana morphotypes with plasminogen. Parasitol Res 88: 972-980.

Carneiro CR, Postol E, Nomizo R, Reis LF, Brentani RR 2004. Identification of enolase as a laminin-binding protein on the surface of Staphylococcus aureus. Microbes Infect 6: 604-608.

Cutler JE 1991. Putative virulence factors of Candida albicans. Annu Rev Microbiol 45: 187-218.

Derbise A, Song YP, Parikh S, Fischetti VA, Pancholi V 2004. Role of the C-terminal lysine residues of streptococcal surface enolase in Glu- and Lys-plasminogen-binding activities of group A streptococci. Infect Immun 72: 94-105.

Dinis M, Tavares D, Veiga-Malta I, Fonseca AJ, Andrade EB, Trigo G, Ribeiro A, Videira A, Cabrita AM, Ferreira P 2009. Oral therapeutic vaccination with Streptococcus sobrinus recombinant enolase confers protection against dental caries in rats. $J$ Infect Dis 199: 116-123.

Eberhard T, Kronvall G, Ullberg M 1999. Surface bound plasmin promotes migration of Streptococcus pneumoniae through reconstituted basement membranes. Microb Pathog 26: 175-181.

Ehinger S, Schubert WD, Bergmann S, Hammerschmidt S, Heinz DW 2004. Plasmin(ogen)-binding alpha-enolase from Streptococcus pneumonia: crystal structure and evolution of plasmin(ogen)binding sites. J Mol Biol 343: 997-1005.

Ghosh A, Edwards MJ, Jacobs-Lorena M 2000. The journey of malaria parasite in the mosquito: hopes for the new century. Parasitol Today 16: 196-201.

Hannaert V, Brinkmann H, Nowitzki U, Lee JA, Albert MA, Sensen CW, Gaasterland T, Müller M, Michels P, Martin W 2000. Enolase from Trypanosoma brucei, from the amitochondriate protist Mastigamoeba balamuthi, and from the chloroplast and cytosol of Euglena gracilis: pieces in the evolutionary puzzle of the eukaryotic glycolytic pathway. Mol Biol Evol 17: 989-1000.

Hotez P, Ottesen E, Fenwick A, Molyneux D 2006. The neglected tropical diseases: the ancient afflictions of stigma and poverty and the prospects for their control and elimination. Adv Exp Med Biol 582: 23-33.

Jolodar A, Fischer P, Bergmann S, Büttner DW, Hammerschmidt S, Brattig NW 2003. Molecular cloning of an alpha-enolase from the human filarial parasite Onchocerca volvulus that binds human plasminogen. Biochim Biophys Acta 1627: 111-120.

Jong AY, Chen SH, Stins MF, Kim KS, Tuan TL, Huang SH 2003. Binding of Candida albicans enolase to plasmin(ogen) results in enhanced invasion of human brain microvascular endothelial cells. J Med Microbiol 52: 615-622.

Keeling PJ 2004. Polymorphic insertions and deletions in parabasalian enolase genes. J Mol Evol 58: 550-556.

Kolberg J, Aase A, Bergmann S, Herstad TK, Rødal G, Frank R, Rohde M, Hammerschmidt S 2006. Streptococcus pneumoniae enolase is important for plasminogen binding despite low abundance of enolase protein on the bacterial cell surface. Microbiology 152: 1307-1317.

López-Villar E, Monteoliva L, Larsen MR, Sachon E, Shabaz M, Pardo M, Pla J, Gil C, Roepstorff P, Nombela C 2006. Genetic and proteomic evidences support the localization of yeast enolase in the cell surface. Proteomics 6 (Suppl. 1): S107-118.

Lottenberg R, Minning-Wenz D, Boyle MD 1994. Capturing host plasmin(ogen): a common mechanism for invasive pathogens? Trends Microbiol 2: 20-24.

Maldonado J, Marina C, Puig J, Maizo Z, Avlin L 2006. A study of cutaneous lesions caused by Leishmania mexicana in plasminogendeficient mice. Exp Mol Pathol 80: 289-294.

Molyneux DH 2006. Control of human parasitic diseases: context and overview. Adv Parasitol 61: 1-45.

Pal-Bhowmick I, Mehta M, Coppens I, Sharma S, Jarori GK 2007. Protective properties and surface localization of Plasmodium falciparum enolase. Infect Immun 75: 5500-5508.

Pancholi V, Fischetti VA 1998. Alpha-enolase, a novel strong plasmin(ogen) binding protein on the surface of pathogenic streptococci. J Biol Chem 273: 14503-14515.

Pizarro-Cerdá J, Cossart P 2006. Bacterial adhesion and entry into host cells. Cell 124: 715-727.

Ponting CP, Marshall JM, Cederholm-Williams SA 1992. Plasminogen: a structural review. Blood Coagul Fibrinolysis 3: 605-614.

Quiñones W, Peña P, Domingo-Sananes M, Cáceres A, Michels PA, Avilan L, Concepción JL 2007. Leishmania mexicana: molecular cloning and characterization of enolase. Exp Parasitol 116: 241-251. 
Read M, Hicks KE, Sims PF, Hyde JE 1994. Molecular characterisation of the enolase gene from the human malaria parasite Plasmodium falciparum. Evidence for ancestry within a photosynthetic lineage. Eur J Biochem 220: 513-520.

Roth EF Jr, Calvin MC, Max-Audit I, Rosa J, Rosa R 1988. The enzymes of the glycolytic pathway in erythrocytes infected with Plasmodium falciparum malaria parasites. Blood 72: 1922-1925.

Rugemalila JB, Ogundahunsi OA, Stedman TT, Kilama WL 2007. Multilateral initiative on malaria: justification, evolution, achievements, challenges, opportunities, and future plans. Am J Trop Med Hyg 77 (Suppl. 6): 296-302.

Sato K, Kano S, Matsumoto Y, Glanarongran R, Krudsood S, Looareesuwan S, Aikawa M, Suzuki M 2000. Application of yeast enolase as antigen for immunodiagnosis of malaria. Southeast Asian J Trop Med Public Health 31 (Suppl. 1): 79-84.

Shao L, Devenport M, Jacobs-Lorena M 2001. The peritrophic matrix of hematophagous insects. Arch Insect Biochem Physiol 47: 119-125.

Snow RW, Guerra CA, Noor AM, Myint HY, Hay SI 2005. The global distribution of clinical episodes of Plasmodium falciparum malaria. Nature 434: 214-217.
Trottier S, Higgins R, Brochu G, Gottschalk M 1991. A case of human endocarditis due to Streptococcus suis in North-America. Rev Infect Dis 13: 1251-1252.

Virkola R, Lähteenmäki K, Eberhard T, Kuusela P, van Alphen L, Ullberg M, Korhonen TK 1996. Interaction of Haemophilus influenzae with the mammalian extracellular matrix. J Infect Dis 173: $1137-1147$

Vora HK, Shaik FR, Pal-Bhowmick I, Mout R, Jarori GK 2009. Effect of deletion of a plant like pentapeptide insert on kinetic, structural and immunological properties of enolase from Plasmodium falciparum. Arch Biochem Biophys 485: 128-138.

Wheat LJ, White AC, Norden C 1985. Serological diagnosis of Staphylococcus aureus osteomyelitis. J Clin Microbiol 21: 764-767.

Zhang A, Chen B, Mu X, Li R, Zheng P, Zhao Y, Chen H, Jin M 2009. Identification and characterization of a novel protective antigen, enolase of Streptococcus suis serotype 2. Vaccine 27: 1348-1353.

Zieler H, Garon CF, Fischer ER, Shahabuddin M 2000. A tubular network associated with the brush-border surface of the Aedes aegypti midgut: implications for pathogen transmission by mosquitoes. J Exp Biol 203: 1599-1611. 